\title{
Transposición de grandes vasos y una rara asociación extracardíaca que cuestionará cuál es la mejor conducta a seguir
}

\author{
Karen Condori Alvino*
}

Carlos Álvarez Murillo**

* Cardióloga Pediatra. Instituto Nacional de Salud del Niño de San Borja (INSN-SB). Lima. Perú

** Cardiólogo Pediatra. Director adjunto del Instituto Nacional de Salud del Niño de San Borja (INSN-SB). Lima. Perú

\section{Correspondencia}

Karen Condori Alvino

kcondori@insnsb.gob.pe

\section{Recibido: 07/01/2020 \\ Aceptado: 28/05/2020 \\ En línea: 31/07/2020}

Citar como: Condori-Alvino K, Álvarez-Murillo C. Transposición de grandes vasos y una rara asociación extracardíaca que cuestionará cuál es la mejor conducta a seguir. Rev Ecocar Pract (RETIC). 2020 (Jul); 3 (2): 11-13. doi: 10.37615/retic.v3n2a4.

Cite this as: Condori-Alvino K, Álvarez-Murillo C. Transposition of great arteries and a rare extracardiac association, that will question what is the best conduct to follow. Rev Ecocar Pract (RETIC). 2020 (Jul); 3 (2): 11-13. doi: 10.37615/retic.v3n2a4.

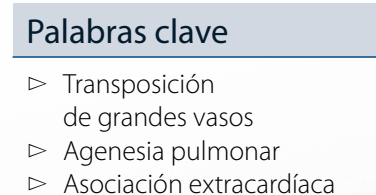

$\triangleright$ Asociación extracardíaca

Keywords

$\triangleright$ Transposition of great arteries

$\triangleright$ Pulmonary agenesis

$\triangleright$ Extracardiac association

\section{Presentación del caso}

Paciente de 3 días de vida procedente de un hospital maternoinfantil, con antecedente de tercera gestación, con controles prenatales normales, nace por cesárea iterativa. Edad gestacional 40 semanas, peso $3,550 \mathrm{~kg}$, talla $49 \mathrm{~cm}$ y Apgar 7-9.

A las horas de vida presenta distrés respiratorio y cianosis perioral, con saturación de oxígeno del $75 \%$, por lo que se realiza una radiografía de tórax (Figura 1) con velamiento de hemitórax izquierdo sin imagen típica de patología cardíaca.

Se le realiza también una ecocardiografía donde se diagnostica transposición de grandes vasos (D-TGV) con comunicación interventricular (CIV) y comunicación interauricular (CIA) asociadas, y sospecha de atelectasia masiva izquierda. Se inicia tratamiento con prostaglandinas y es transferido al instituto de referencia.
Ingresa ventilando espontáneamente. Se confirma el diagnóstico cardíaco: D-TGV, CIV de $4 \mathrm{~mm}$, CIA ostium secundum de $8 \mathrm{~mm}$ y persistencia de conducto arterioso (PCA) de $5 \mathrm{~mm}$.

Se realiza una angiotomografía para evaluar la patología cardíaca asociada (Figura 2 a Figura 5), diagnosticándose aplasia pulmonar izquierda. Se mantiene el tratamiento con prostaglandinas por 10 días, ya que el tamaño de la CIA y la PCA eran amplios para asegurar mezcla y es dado de alta a los 28 días. Se mantienen controles ambulatorios hasta los 2 meses de edad.

Posteriormente se le admitió para cirugía cardíaca programada con apoyo de misión médica extranjera (CUIDAM). Se le realizó switch arterial, cierre de la CIV y ligadura y sección del conducto arterioso. El tiempo de circulación extracorpórea (CEC) fue de 2 horas y 30 minutos, y el tiempo de clampado aórtico de 1 hora y 27 minutos. El resultado quirúrgico fue bueno y la evolución postoperatoria satisfactoria.

Fue dado de alta y sigue controles médicos periódicos. 


\section{RETIC}

Revista de ecocardiografía

práctica y otras técnicas de imagen cardíaca

\section{Estudio por imagen}

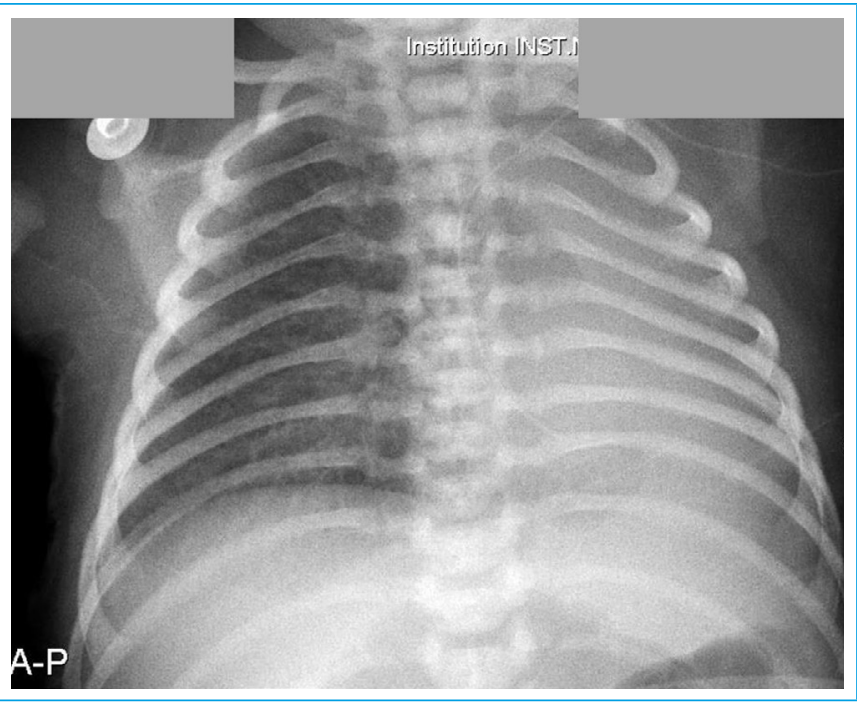

Figura 1. Radiografía de tórax que muestra desviación de silueta cardíaca y opacidad difusa en hemitórax izquierdo

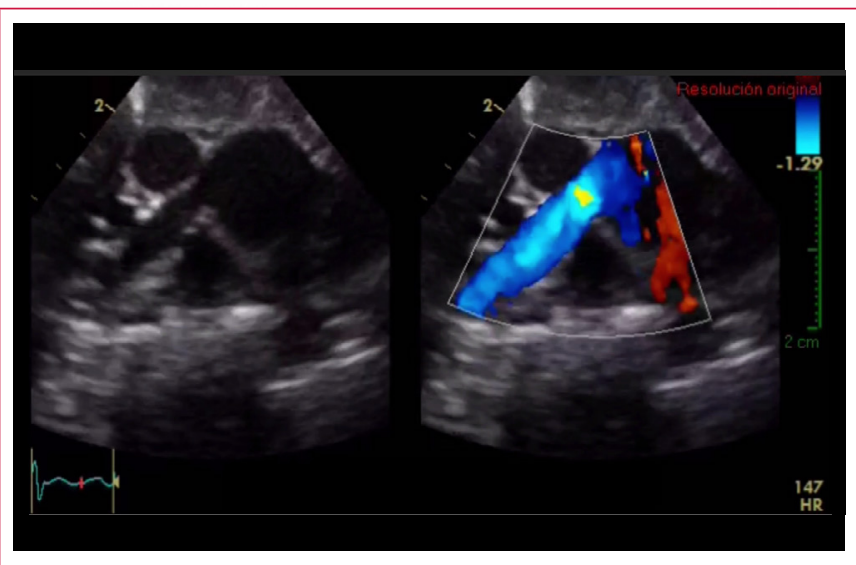

Vídeo 1. Imagen en eje corto que muestra bifurcación pulmonar con arteria pulmonar principal de buen tamaño, la rama izquierda tiene flujo diastólico invertido (rojo)

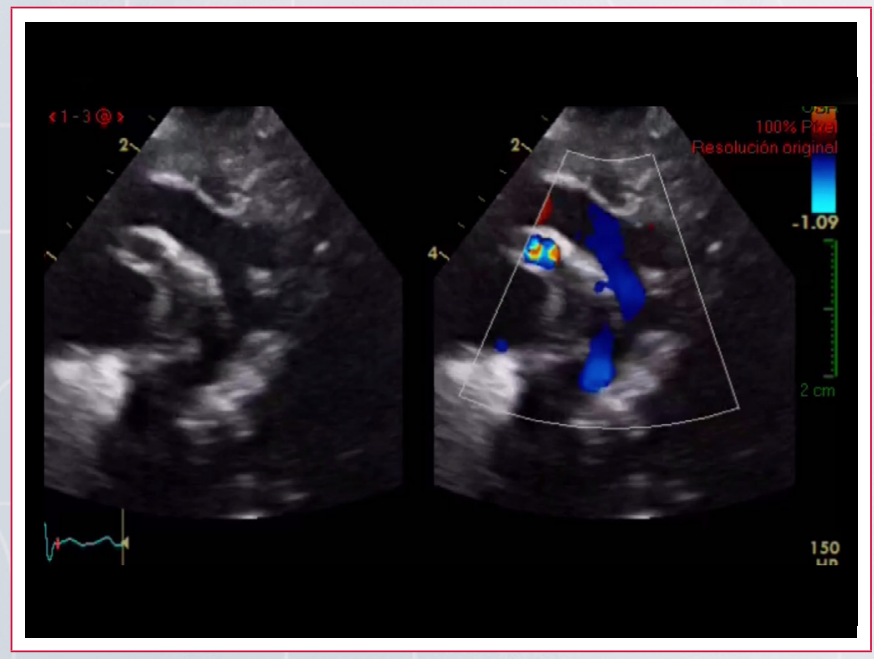

Vídeo 2. Imagen supraesternal. Ductus arterioso persistente amplio con flujo sistólico de derecha a izquierda (azul) y flujo diastólico retrógrado (rojo)

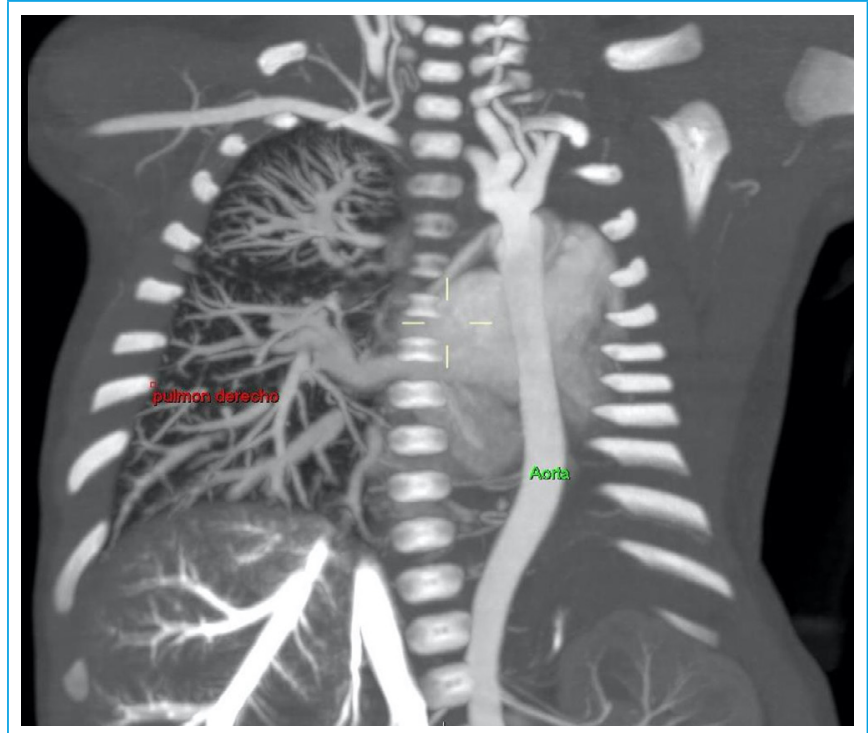

Figura 2. La tomografía muestra escaso tejido pulmonar izquierdo, además de componente atelectásico
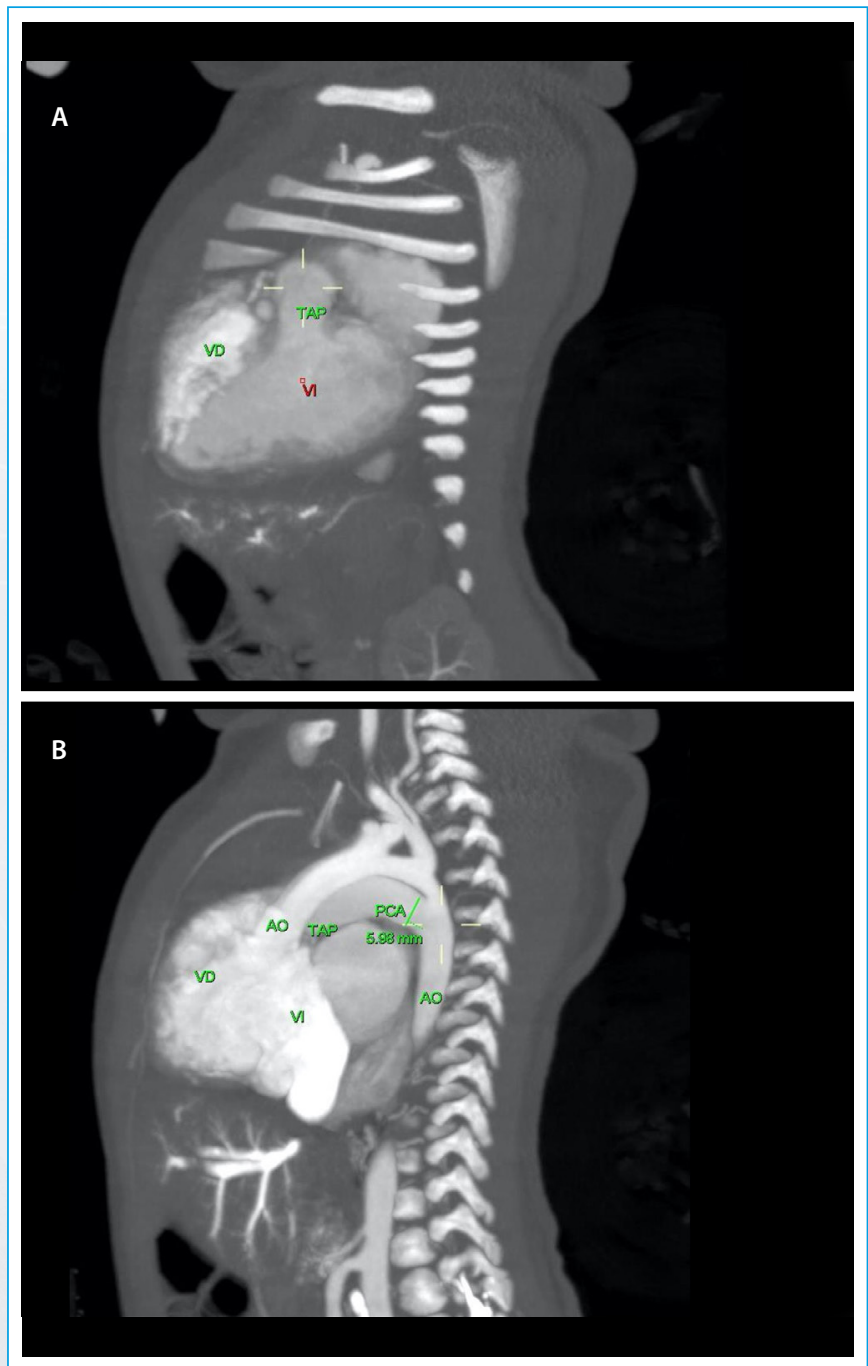

Figura 3. A: se evidencia la conexión ventriculoarterial discordante; B: vista sagital que muestra la transposición de los grandes vasos y el ductus arterioso amplio (AO: aorta; PCA: conducto arterioso persistente; TAP: tronco arteria pulmonar; VD: ventrículo derecho; VI: ventrículo izquierdo) 


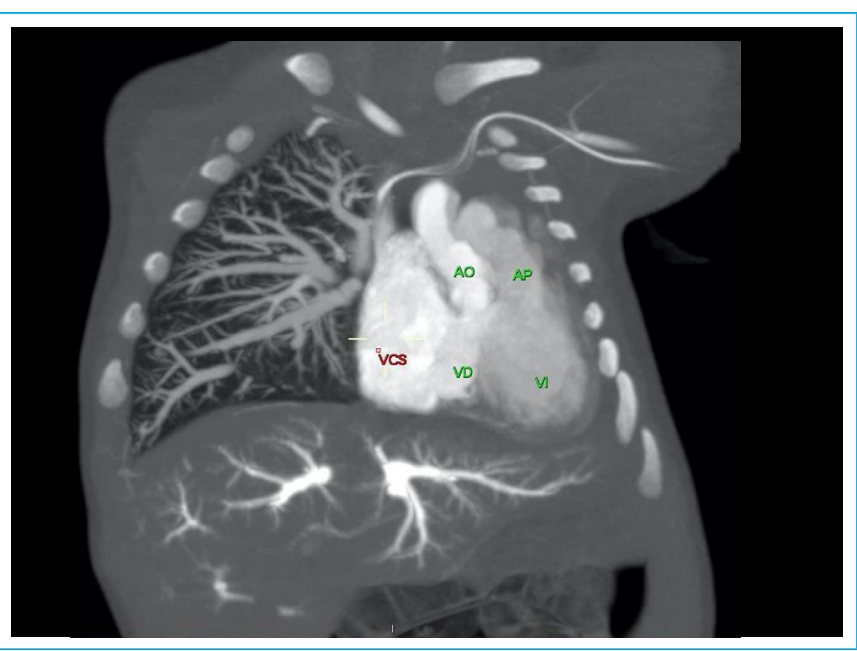

Figura 4. Vista coronal en donde se observa la discordancia ventriculoarterial y la silueta cardíaca ocupando el hemitórax izquierdo por la agenesia pulmonar (AO: aorta; AP: arteria pulmonar; VCS: vena cava superior; VD: ventrículo derecho; VI: ventrículo izquierdo)

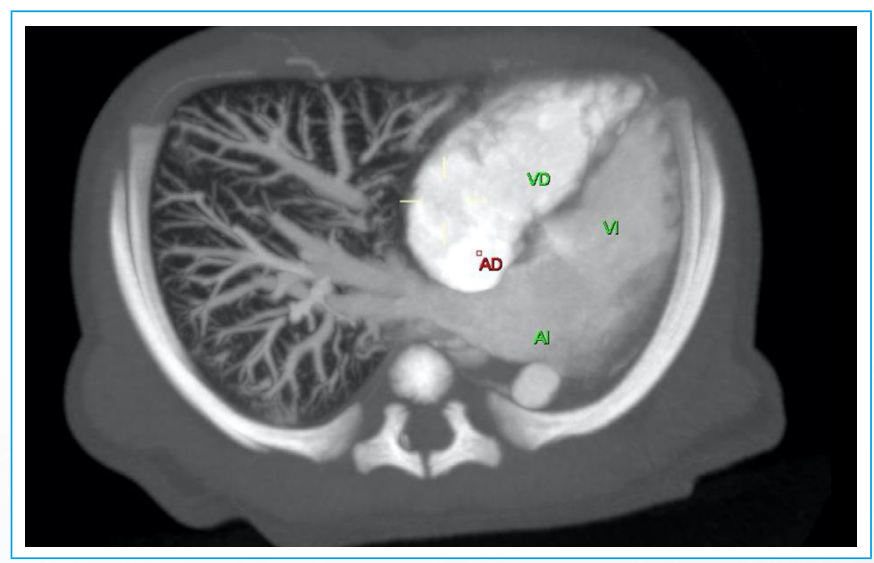

Figura 5. Vista axial que muestra discordancia ventriculoarterial y la silueta cardíaca ocupando hemitórax izquierdo por la agenesia pulmonar (AD: aurícula derecha; Al: aurícula izquierda; VD: ventrículo derecho; VI: ventrículo izquierdo)

\section{Discusión}

La transposición de grandes vasos es una de las cardiopatías congénitas más comunes y severas, pero también una de las más misteriosas. Tiene una prevalencia de 3,54/10.000 nacimientos en Europa y es la cuarta cardiopatía compleja más frecuente ${ }^{(1)}$. Representa el $5 \%$ de todas las cardiopatías congénitas y el $34 \%$ de los defectos conotroncales con situs solitus. Sin tratamiento, el $30 \%$ de los casos muere antes del primer mes y el $90 \%$ antes del primer año(2). En los últimos años se han realizado grandes mejoras en el diagnóstico, así como en el tratamiento médico y quirúrgico de esta cardiopatía congénita. Como consecuencia, en la actualidad, la supervivencia general de estos pacientes es significativamente mejor ${ }^{(3)}$.

La agenesia pulmonar es poco común. Consiste en la ausencia total o en la hipoplasia severa de uno o de ambos pulmones. Fue descrita por primera vez en 1673 por De Pozze, quien la observó de manera incidental durante la autopsia de una joven. La primera documentación de un caso se realizó en 1887 por Haberlein. La frecuencia aproximada de la agenesia pulmonar es de 1/15.000 nacidos vivos ${ }^{(4)}$.

No se ha encontrado más información de esta asociación en la literatura, si bien es cierto que la D-TGV es la cardiopatía cianótica más frecuente en el neo- nato y con una adecuada anatomía valvular de los grandes vasos y las coronarias, y una adecuada masa ventricular, una reparación quirúrgica temprana marcará la diferencia.

En un estudio en México se asoció un $46 \%$ de malformaciones extracardíacas a D-TGV y dentro del sistema respiratorio sólo se asoció bronquio accesorio izquierdo e isomerismo pulmonar derecho(5).

El pronóstico es mejor cuando la agenesia pulmonar es unilateral a la izquierda y cuando están ausentes malformaciones extracardíacas. Sin embargo, en el paciente de este caso la asociación importante con agenesia pulmonar izquierda no fue impedimento para tomar la decisión de programarlo para el switch arterial. Tuvo un postoperatorio óptimo, con buen resultado cardíaco, y fue dado de alta con controles ambulatorios por los servicios de Cardiología y Neumología.

\section{Conclusiones}

La transposición de grandes vasos es una de las cardiopatías congénitas más comunes y severas pero también una de las más misteriosas. En los últimos años, se han realizado grandes mejoras en el diagnóstico, así como en su tratamiento médico y quirúrgico. Como consecuencia, en la actualidad, la supervivencia general de estos pacientes es significativamente mejor.

El caso que hemos mostrado es muy infrecuente, las imágenes mostradas no eran usuales, y es que una rara asociación extracardíaca (agenesia pulmonar izquierda) cambió la conducta diagnóstico-terapéutica.

\section{Ideas para recordar}

- La transposición completa de grandes vasos es una de las cardiopatías cianóticas más frecuentes en el recién nacido.

- La forma de presentación clínica varía de acuerdo con las lesiones asociadas, CIV, CIA, ductus y si tiene o no estenosis pulmonar.

- La ecocardiografía es la piedra angular del diagnóstico, incluso desde la vida fetal.

- La angiotomografía complementa muy bien los hallazgos ecocardiográficos.

- La agenesia pulmonar es una asociación extracardíaca muy rara en la D-TGV, casi no descrita, y si es izquierda tiene mejor pronóstico.

\section{Bibliografía}

1. Pexieder T, Blanc O, Pelouch V, et al. Late fetal development of retinoic acidinduced transposition of great arteries: morphology, physiology and biochemistry. En: Clark EB, Markwald RR, Takao A (ed.). Developmental mechanism of heart disease. New York. Futura Publishing Company, 1995; 297-307.

2. Ferencz C, Brenner Jl, Loffredo C, et al. Transposition of great arteries: etiologic distinctions of outflow tract defects in a case-control study of risk factors. En: Clark EB, Markwald RR, Takao A (ed.). Developmental mechanism of heart disease. New York. Futura Publishing Company, 1995; 639-653.

3. Mair DD, Ritter DG, Ongley PA, Helmholz HF. Hemodynamics and evaluation for surgery of patients with complete transposition of the great arteries and ventricular septal defect. Am J Cardiol 1971; 28 (6): 632-640.

4. Manohar VM, Shivanna DN, Ramesh, Vemgal P. Left pulmonary agenesis with single atrium simulating cardiac type of total anomalous pulmonary venous connection. Ind J Thorac Cardiovasc Surg 2008; 24: 180-183.

5. Quispe-Susara EN, Durán-Padilla MA, Ramón-García G. Malformaciones cardiacas y extracardíacas asociadas a transposición de grandes vasos. Revisión de 15 casos. Rev Med Hosp Gen Mex 2006; 69 (3): 144-148. 